\title{
Thérapie de conversion
}

Peter Schulthess

Il n'y a pas si longtemps encore, l'homosexualité était considérée par l'OMS comme une maladie dont les causes psychologiques pouvaient être traitées. Ce n'est qu'en 1992 qu'elle a finalement été retirée de la liste des maladies de la CIM-10. Des articles de presse récents (par exemple dans le Sonntagszeitung du 16 juin 2019) montrent que l'idée qu'il s'agit d'une maladie guérissable est toujours présente en Allemagne.

Sous le nom de thérapie de conversion, on a connu une procédure qui promet de guérir l'homosexualité et de la transformer en hétérosexualité à l'aide de techniques comportementales. On l'appelle aussi parfois thérapie réparatrice. Cette forme de thérapie a été développée par trois psychologues, Joseph Nicolosi, Mark A. Yarhouse (professeur d'université à l'Evangelical Regent University aux Etats-Unis) et Richard Cohen (qui a été privé de sa licence de thérapie en raison de violations de plusieurs règles éthiques). Les tentatives de "repolir " thérapeutiquement les homosexuels sont soutenues par les cercles de l'Église évangélique, qui considèrent que les écarts par rapport à l'hétérosexualité ne sont pas en harmonie avec leur vision de l'homme et en contradiction avec la volonté de Dieu. Le Vatican n'a précisé que le 27 août 2018 que l'homosexualité nétait pas une maladie et que les thérapies de conversion nétaient donc pas préconisées.

En Allemagne, la thérapie de conversion est toujours propagée par l'« Institut allemand pour la jeunesse et la société » ("Deutschen Institut für Jugend und Gesellschaft ») et par «Wüstenstrom ", un mouvement chrétien évangélique qui offre des conseils, des séminaires et des groupes d'entraide pour les personnes qui vivent les relations, leur sexualité ou leur identité en tant que femmes ou hommes en situation conflictuelle. Le but de ces thérapies spirituellement accompagnées est de trouver une identité hétérosexuelle. Wüstenstrom propose une formation de thérapeute en conversion (h/f). Même en Suisse, la thérapie de conversion est toujours appliquée par Wüstenstrom, dans l'entourage de l'église libre de Chrischona.

La thérapie de conversion ne peut pas être comprise comme une psychothérapie lege artis. Cette procédure n'est reconnue par aucune organisation professionnelle psychothérapeutique et il n'existe aucune preuve de son efficacité. Cependant, l'American Psychological Association (APA), par exemple, la considère comme nuisible et recommande aux professionnels de la santé mentale de s'abstenir d'expliquer à leurs clients que l'orientation sexuelle peut être modifiée par la thérapie.

La thérapie de conversion, en conjonction avec la pensée évangélique dans le but de réorientation sexuelle, est une violation des directives éthiques de toutes les associations professionnelles sérieuses. Les valeurs des thérapeutes ne doivent pas être transférées aux patients. Bien que les questions de l'insécurité dans sa propre orientation psychosexuelle devraient et pourraient faire l'objet de psychothérapies - même pour les adolescents - le résultat devrait toujours être ouvert, afin que le patient puisse découvrir et consolider sa propre orientation. Introduire ses propres jugements et les transmettre aux patients est un abus de pouvoir et une violation des droits de la personnalité des patients (h/f).

Aucun psychothérapeute sérieux, ni aucune psychothérapeute sérieuse, n'acceptera une affectation thérapeutique pour repolir sexuellement un patient, même pas à sa demande. Au contraire, il ou elle découvrira ce qui pousse quelqu'un à vouloir être guéri de son homosexualité. Ce faisant, on trouvera des problèmes de discrimination et de souffrance liés au fait de ne pas être hétérosexuel qui doivent être réglés.

Lété dernier, un psychiatre (de l'Église libre Chrischona) de Suisse centrale a proposé une thérapie de conversion pour les jeunes aux frais de l'assurance maladie. Du fait que l'homosexualité n'est pas une maladie, la caisse d'assurance maladie n'a certainement pas à payer pour cela. Le médecin serait poussé dans ce cas à faire d'autres diagnostics et cacher ainsi le fait que lobjectif thérapeutique est la "guérison " de l'homosexualité, commettant ainsi une fraude à l'assurance. Il appartient à la FMH et à la direction de la santé compétente, qui délivre les autorisations de pratique, de tenir ce médecin responsable, le cas échéant également les caisses d'assurance maladie, si les prestations correspondantes ont effectivement été facturées.

Lexemple montre qu'il n'est malheureusement pas exclu que même des psychothérapeutes (h/f) ou des psychiatres (h/f) sérieusement formé(e)s ne soient pas à l'abri de voir l'homosexualité ou d'autres orientations sécartant de l'hétérosexualité comme une pathologie et souhaitent la traiter à cause de leurs visions de l'homme imprégnées par l'évangélisme.

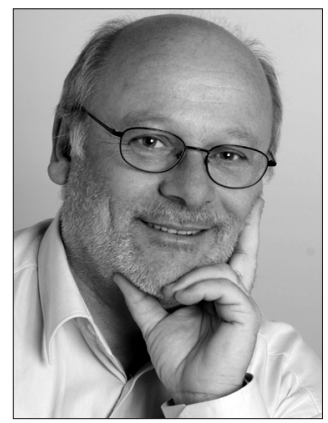


Au Parlement européen en 2018, une nette majorité de tous les partis politiques sest opposée à une initiative visant à introduire une interdiction légale des thérapies pour « guérir » l'homosexualité. En février 2019, le ministre allemand de la Santé, Jens Spahn, a annoncé une interdiction légale des thérapies de conversion en Allemagne, à la suite de quoi les gouvernements de quatre États allemands ont annoncé en avril 2019 un projet de loi correspondant pour leurs propres régions (Länder). En Suisse, la Conseillère natio- nale Rosmarie Quadranti a présenté une motion en ce sens, que le Conseil fédéral recommande toutefois au Parlement de rejeter.

L'ASP s'est clairement positionnée dans ce domaine avec deux communiqués de presse à ce jour (https://psychotherapie.ch/wsp/de/aktuell/ medien). Lencadré ci-dessous contient le communiqué de presse de l'ASP du 5 septembre 2019.

Peter Schulthess est membre du comité de l'ASP. 
Nous nous réjouissons que le Conseil fédéral reconnaisse également que « la thérapie visant à changer l'orientation homosexuelle doit être rejetée d'un point de vue humain, professionnel et juridique ». L'homosexualité n'est pas une maladie, donc il n'y a rien à traiter.

Dans ses observations, le Conseil fédéral méconnaît que les thérapies de conversion généralement ne sont pas proposées ou pratiquées par des psychothérapeutes (h/f) ou psychiatres (h/f) reconnu(e) s. S'ils le sont, c'est bien par des thérapeutes qui appartiennent eux-mêmes à un groupe religieux évangélique qui, contrairement à l'état actuel de la technique et de la science, considèrent encore l'homosexualité comme une maladie ou un trouble du développement mental qu'ils voudraient guérir. Ils commettent ainsi une faute professionnelle et violez l'éthique professionnelle. Et peut-être qu'ils manquent de formation continue et n'ont pas remarqué que l'homosexualité a depuis longtemps été retirée des systèmes internationaux de classification des troubles mentaux.

Les médecins et psychothérapeutes (h/f) psychologues membres d'une association professionnelle peuvent être rendus responsables par les personnes lésées et " soignées " à travers de comités professionnels ou commissions éthiques de ces associations ou par l'intermédiaire des directions cantonales des affaires sanitaires et de la santé des cantons. Il convient toutefois de noter que le législateur a expressément indiqué dans la LPsy qu'un(e) psychothérapeute (h/F) reconnu(e) par le gouvernement fédéral n'est pas tenu(e) d'appartenir à une association professionnelle. Cela signifie qu'il y a de plus en plus de psychothérapeutes (h/f) qui n'appartiennent à aucune association professionnelle, ce qui signifie qu'aucun comité d'éthique ou d'éthique n'est responsable. Ni la Confédération ni les cantons ne veulent revoir le devoir de formation continue inscrite dans la même loi et laissent cette tâche aux associations, qui ne peuvent toutefois le faire que pour leurs membres.

Plus grave encore, le Conseil fédéral ne tient pas compte du fait que la thérapie de conversion est généralement proposée et pratiquée par des «thérapeutes (h/F) chrétiens » sans formation qualifiée en psychothérapie. Il existe un nombre croissant de formations chrétiennes en thérapie de conversion dans l'environnement des églises libres comme l'église Chrischona et d'autres organisations évangéliques, dont les diplômés ne sont soumis ni à la LPMéd ni à la LPsy, n'appartiennent pas à une association professionnelle correspondante et ne nécessitent pas d'autorisation de pratique cantonal pour leur activité. Ces " thérapeutes (h/F) " travaillent dans un vide juridique et justifient leur travail par une interprétation évangélique de la Bible, selon laquelle l'homosexualité signifie une anomalie qui doit être traitée. Ces « thérapeutes (h/F) » opèrent dans un vide juridique et nuisent aux homosexuels croyants (dont la plupart sont des enfants de familles évangéliques) sans avoir à craindre les conséquences juridiques. Les adolescents et leurs parents ne se rendent pas compte que ces « thérapeutes » ne sont pas des psychothérapeutes (h/F) avec des qualifications professionnelles.

Si le Conseil fédéral veut sérieusement rejeter de telles thérapies également d'un point de vue juridique, il doit créer une disposition pénale correspondante qui érige en infraction punissable la thérapie de conversion et les autres procédures visant à renverser l'orientation sexuelle. Ce n'est qu'ainsi que l'on pourra combler le manque de compétences des psychothérapeutes (h/F) et des psychiatres (h/f) qui ne font pas partie d'une association et du vaste champ des conseillers et des thérapeutes chrétiens.

Pour l'ASP, il n'est pas clair comment le Conseil fédéral parvient à la conclusion qu'il soit impossible de consacrer une telle interdiction dans le droit suisse. Après tout, il s'agit d'une violation grave et délibérée de l'intégrité personnelle des personnes concernées, protégée par la Constitution, d'une discrimination, d'une violation des droits humains et d'une forme d'exercice de la violence. Il ne suffit pas de souligner que de tels cas peuvent être signalés à la KESB, qui ne dispose pas non plus d'une base juridique pour ses actions sans interdiction. Une norme juridique claire affecterait d'une part tous les thérapeutes imaginables en tant qu'auteurs possibles d'un délit et faciliterait d'autre part le travail des commisions d'éthique des associations ainsi que des directions générales cantonales de la santé.

Nous espérons que le Parlement discutera sur la question plus en profondeur que le Conseil fédéral et qu'il soumettra néanmoins la motion. 